\title{
Research Paper: The Lee Silverman Voice Treatment Improves Vocal Loudness in Early Persian-speaking Patients With Parkinson's Disease
}

\author{
Younes Amiri Shavaki ${ }^{1}$ (D), Arezoo Saffarian ${ }^{1 *}$ (D), Gholam Ali Shahidi² ${ }^{\text {DD }}$, Mohammad Reza Keyhani ${ }^{3}$ (D) \\ 1. Department of Speech and Language Pathology, School of Rehabilitation Sciences, Iran University of Medical Sciences, Tehran, Iran. \\ 2. Movement Disorders Clinic, Hazrat Rasool Hospital, Iran University of Medical Sciences, Tehran, Iran. \\ 3. Department of Basic Sciences, School of Rehabilitation Sciences, Iran University of Medical Sciences, Tehran, Iran.
}

\begin{tabular}{|c|c|}
\hline $\begin{array}{l}\text { Use your device to scan } \\
\text { and read the article online }\end{array}$ & Citation: Amiri Shavaki Y, Saffarian A, Shahidi GA, Keyhani MR. The Lee Silverman Voice Treatment Improves Vocal \\
\hline 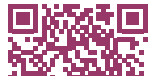 & $\begin{array}{l}\text { Loudness in Early Persian-speaking Patients With Parkinson's Disease. Iranian Rehabilitation Journal. 2021; 19(1):75-84. http:// } \\
\text { dx.doi.org/10.32598/irj.19.1.1107.1 }\end{array}$ \\
\hline 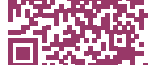 & doi'http://dx.doi.org/10.32598/irj.19.1.1107.1 \\
\hline
\end{tabular}

Article info:

Received: 01 Apr 2020

Accepted: 30 Mar 2021

Available Online: 01 Mar 2021

Keywords:

Voice, Treatment, Loudness, Voice disorders, Parkinson disease

\begin{abstract}
Objectives: In the early stages, most Parkinson's patients display some degrees of voice and speech disorders. Speech disorders can negatively affect individuals' social interactions and life; thus, treatment plans for the disorders in the early stages might be further beneficial than the late stages. Therefore, we aimed to investigate the effects of an intervention on Persianspeaking patients with early mild Parkinson's Disease (PD). The intervention was the Lee Silverman Voice Treatment (LSVT).

Methods: This was an interventional study and included 23 patients with PD. The study participants were randomly assigned into one of two groups; 13 patients in the intervention group (PD-I), and 10 in No-Intervention (PD-NI) group. Normal Persons (NP) or the control group included 13 healthy participants who were neurologically normal and presented no speech and voice disorders. Besides, they were matched with the PD-I group concerning education, age, gender.

Results: There was a significant impairment in Maximum Phonation Time (MPT), vocal loudness in vowel $/ \mathrm{a} /$, in reading, and during a monologue speech in the PD group, compared with the NP group $(\mathrm{P} \leq 0.001)$. The LSVT resulted in a significant improvement in all measures in the PD-I group $(\mathrm{P} \leq 0.001)$; however, no significant change was observed between the PD-I and PD-NI groups' scores in first assessment ( $P \geq 0.201)$. In contrast, the PD-NI group's results in all measurements of final assessment were significantly lower than those in the PD-I $(\mathrm{P} \leq 0.001)$ and $\mathrm{NP}(\mathrm{P} \leq 0.001)$ groups. The MPT also exhibited a considerable improvement in the PD-I group, compared with the controls.
\end{abstract}

Discussion: The LSVT, as a therapeutic technique based on the motor learning theory was beneficial in improving the vocal loudness of Persian-speaking patients with PD in the early stages of their disease.

\footnotetext{
"Corresponding Author:

Arezoo Saffarian, PhD.

Address: Department of Speech and Language Pathology, School of Rehabilitation Sciences, Iran University of Medical Sciences; Madadkaran St.,

Shahid Shah-Nazari St., Mother Square, Mirdamad Blvd, Tehran, Iran.

Tel: +98 (21) 22228051

E-mail:asaffarian_slp@yahoo.com
} 


\section{Highlights}

- The explored patients in the early stage of Parkinson's disease, compared with the neurologically normal control group, had significant impairment in Maximum Phonation Time (MPT), vocal loudness in vowel /a/, reading, and during a monologue speech.

- The presented LSVT significantly improved vocal loudness in Persian-speaking Parkinson's patients who were in the early stages.

- There was a considerable increase in the MPT of the vowel /a/ in the studied patients in the PD group with the intervention (PD-I), even a little more than that in the healthy controls.

\section{Plain Language Summary}

Speech and voice disorders are observed in almost $90 \%$ of the Persian-speaking patients with Parkinson's Disease (PD) during their course of illness. Reduced vocal loudness is among the vocal characteristics of these patients, which negatively affects social interactions and quality of life in them. A method for treating speech and vocal difficulties in patients with PD is the Lee Silverman Voice Treatment (LSVT LOUD), i.e., used in other countries. In this study, we used LSVT for treating early-stage Parkinson's patients in Iran. Accordingly, LSVT has led to an increase in vocal loudness in the speech of the explored patients with PD.

\section{Introduction}

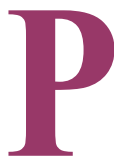

arkinson's Disease (PD) usually occurs in aging groups and is progressive. This neurological disorder seems to be correlated with reduced dopaminergic neurons. The main observable symptom is rest tremor; two other main symptoms are bradykinesia and motion rigidity; the other disabling feature is postural instability [1]. Dysarthria, as a motor speech and voice disorder is observed in almost $90 \%$ of patients with PD during their course of illness [2]. Reduced voice pitch inflections or monotone speech, reduced vocal loudness, the imprecise articulation of the consonants, hoarseness, and breathy voice is also observed in patients with PD; these symptoms often lead to reduced speech intelligibility [3]. Furthermore, patients with PD lack a proper perception of their voice. They are unaware that their loudness is reduced, leading to an inability to self-controlling their voice; this unawareness is due to sensory processing deficits in patients with PD. They also encounter deficits in internal cueing [4], and even the perception of emotions through the auditory channel [5]. These speech disorders can negatively affect social interactions and quality of life in patients with PD [6].

Among diverse therapeutic approaches applied in mitigating voice and speech disorders in patients with PD, speech therapy is among the most effective noninvasive approaches [7]. Speech therapies focused on speed and articulation to improve the symptoms of the disease have presented limited effectiveness; their long-term effects have been unsuccessful [4]. A behavioral method for treating speech and vocal difficulties in patients with PD is the Lee Silverman Voice Treatment (LSVT LOUD) developed by Ramig and associates [8].

The LSVT differs from the conventional types of speech therapy. The LSVT includes 3 specific features, as follows: voice is the target of the intervention; it is a high-effort and intensive therapy; it emphasizes calibration, leading to the generalization and long-term effects of the intervention [4]. The LSVT is a behavioral intervention approach; accordingly, Randomized Controlled Trials (RCTs) and other evidence revealed its effectiveness to improve loudness and enhance other vocal features up to several months after the intervention [9-12]. The intervention focuses on high-effort intensive vocal activities, especially when accompanied by proprioceptive feedback and auditory-vocal self-monitoring; thus, it helps to rescale the speech output amplitude. Focusing on self-monitoring, i.e., damaged due to sensory processing deficits in the $\mathrm{PD}$, is an essential part of the intervention plan [4].

The LSVT can modify the vocal loudness, Sound Pressure Level (SPL), vocal fold adduction, voice quality, articulation, Voice Handicap Index (VHI), and speech intelligibility in patients with PD [10,11,13-15]. Neu- 
roimaging data highlighted the early evidence of neural plasticity as a result of LSVT [16].

In the majority of past research, the LSVT was applied in patients with PD of mild to moderate severity, or the average disease severity of more than grade 2, according to the Hoehn and Yahr scale [16]. Few studies, however, reported the effectiveness of the LSVT in the early stages in mitigating the progression of voice and speech symptoms in patients with PD [11]. The LSVT could be more efficient in decreasing the incidence or progression of verbal symptoms at the early stages. It presumably is addressed as the lower prevalence of the concurrent accompanying cognitive or psychological issues, i.e., depression, fatigue, or dementia [4].

PD is common in the elderly [1]; thus, it severely affects their mental health and quality of life. This is the case with their families, too. These are often influenced by the loss of conversational and communicative abilities. Furthermore, such constraints become aggravated in the progressed stages of the disease. Therefore, using successful rehabilitation methods is crucial in blunting speech and voice problems at the soonest possible. On the other hand, only one research investigated the LSVT impacts on Persian-speaking patients with PD. In that study, researchers studied the effects of LSVT on the Voice Handicap Index (VHI) [15]. Thus, the effects of this intervention on the vocal loudness of early or mild stages of patients with PD without the symptoms of dementia and depression were investigated in this study. We hypothesized that this intervention would improve vocal abilities in the PD group receiving Intervention (PD-I), compared with the PD group without intervention (PD-NI).

\section{Methods}

The present interventional study consisted of the following groups: one: 13 patients with $\mathrm{PD}(\mathrm{Mean} \pm \mathrm{SD}$ age: $56.64 \pm 4.70$ years; 7 females \& 6 males) receiving the LSVT intervention; two: 13 neurologically-healthy individuals as the controls (NP) (Mean \pm SD age: $56.14 \pm 3.30$ years; 7 women $\& 6$ men), who were matched with the intervention group (PD-I); three: 10 patient with PD receiving No-Intervention (PD-NI) (Mean $\pm \mathrm{SD}$ age: $57.82 \pm 3.46$ years; 5 females \& 5 males). These 23 individuals were randomly assigned into the PD-I and PD-NI groups. For the random assignment of them, each subject was initially allocated a code; then, an unaware individual selected the codes per group. The NP group presented no voice problems or speech disorders. The matching criteria were age, gender, and educational lev- el. The exclusion and inclusion criteria of the NP group were the same as the patient groups (Table 1).

The inclusion criteria of the current study included the following: neurologist diagnosed idiopathic cases by valid criteria [17]; mild cases in stage 1 or 2, i.e., determined by Hoehn and Yahr Scale [18]; the idiopathic cases, i.e., diagnosed 1-8 years ago ((Mean \pm SD duration: $4.67 \pm 1.24$ years) [3]; 4. using levodopa medication [13]; before performing any part of the intervention or homework, the study subjects were requested to ensure that the employed sound of the tester could be easily heard [10]; monolingual native Persian speakers; right-handedness [16]; a minimum level of high school education, and complaints about voice and speech by patients or patient relatives [11]. The exclusion criteria included the following: an experience of head trauma, or brain stroke, or surgery [19]; receiving different antiparkinsonian treatment approaches, such as subthalamic nucleus deep brain stimulation (STN-DBS) (except for the consumption of levodopa) [19]; a history of surgery or damage in the larynx [20]; obtaining depression scores above 10 as measured by the Geriatric Depression Scale (GDS) [21] and achieving a general cognitive ability score of $<26$ (the maximum score of the MMSE test equals 30) [22]. During or before the study, the PD-I and PD-NI groups underwent no intervention program by speech-language pathologists [13]. The dose of medications used by patients remained unchanged when the evaluation and intervention were accomplished [11].

All research participants provided an informed consent form. The PD-NI group was suggested to receive the same intervention after the study fulfillment. The intervention was free of charge for them. The methodological procedure insisting on the intervention plan and the speech tasks used for assessments are comprehensively presented below (Figure 1).

The LSVT intervention was performed by one speechlanguage pathologist, certificated by the LSVT Global Inc. The LSVT, with a set of simple tasks, is designed to maximize respiratory and phonatory functions. This method is performed with continuous guidance and stimulation of the patient to produce a loud voice with high effort during sustained phonation and various speech tasks [8]. The LSVT approach includes the following fundamental concepts: focusing on voice (especially producing loud voice); stimulating speech production with high-effort and repetitive items at different times; intensive intervention; increased sensory awareness, and quantifying behaviors. Intervention sessions included the tasks of daily living and a speech hierarchy 
Table 1. The demographic characteristics of the study groups (Mean $\pm \mathrm{SD}$ )

\begin{tabular}{cccccc}
\hline Groups & Age $(\mathbf{y})$ & Education $(\mathbf{y})$ & GDS Score & MMSE Score & Disease Severity (H\&Y) \\
\hline PD-I $(n=13)$ & $56.64 \pm 4.70$ & $15.32 \pm 2.57$ & $2.71 \pm 0.91$ & $28.37 \pm 0.91$ & $1.46 \pm 0.49$ \\
PD-NI $(n=10)$ & $57.82 \pm 3.46$ & $15.87 \pm 1.97$ & $2.60 \pm 0.84$ & $28.10 \pm 0.87$ & $1.20 \pm 0.34$ \\
NP $(n=13)$ & $56.14 \pm 3.30$ & $15.64 \pm 1.82$ & $1.57 \pm 0.71$ & $29.57 \pm 0.64$ & - \\
\hline
\end{tabular}

Iranian Rehabilitation Journal

GDS: Geriatric Depression Scale, MMSE: Mini-Mental State Examination, PD-I: Parkinson's Disease-Intervention, PD-NI: Parkinson's Disease No-Intervention.

[8]. These tasks improve the loudness of voice by the repetitions of vowel ("ah") in a sustained form, exercises on high/low-pitch range, and some phrases with daily living usage. The speech hierarchy can systematically increase communicative functions by further practice with patients for maintaining increased loudness of voice (achieved by daily living tasks) for higher of speech and more complicated speaking situations (e.g. progression from words to daily living conversations) [16]. The explored individuals were advised to produce "louder voice" when conducting different tasks, such as phonation and speaking. Besides, for enhancing self-regulation, they received auditory and visual feedback [10].

The LSVT approach includes 16 one-hour intervention sessions, presented individually in 4 weeks (4 sessions/week). The LSVT was administered for all study patients based on the standard LSVT LOUD protocol by Ramig and associates [8]. The intervention sessions were performed in a quiet place. Before starting the intervention, a speech and language pathologist who was a fluent speaker in Persian and English languages translated the clinical tasks of LSVT into Persian [14].

The speech tasks were recorded one week before the onset of the intervention plan for the intervention groups and one week after finishing the intervention sessions [9]. The recording was conducted in an acoustic room with a background noise level of $<43 \mathrm{~dB}$ [19], using ASUS Cerberus microphone and Praat 5.4 software at the sampling rate of $44.1 \mathrm{KHz}$, and an $8-\mathrm{cm}$ distance between mouth and microphone [14]. The vocal loudness (dB SPL) was obtained by GM1357 sound level meter located at a $30-\mathrm{cm}$ distance of the lips [16]. The time of medication consumption for all research participants was one hour or more before recording. Furthermore, they were in the on-phase of their medication cycle while recording the speech tasks [14]. The recording method for the PDNI and NP groups was the same as the PD-I group; the speech tasks were recorded in 2 periods, i.e., primary and secondary evaluations, concurrent with the PD-I group.
The speech tasks used for all study groups included the following: maximum duration of the sustained phonation of vowel /a/; reading the Grandfather text (it consists of 15 sentences, 13 predicative sentences, 1 interrogative sentence, \& 1 exclamatory sentence; 83 words and 160 syllables with an average syllable per word of 1.93) (This text is prepared to be easily read by low-literacy individuals) [23], and a 25 to 30 seconds of monologue conversational speech based on the individual's choice [10]. To calculate the Maximum Phonation Time (MPT) of the vowel /a/, the study participants were requested to sustain vowel /a/ for as long as possible. Each study participant repeated vowel /a/ 3 times; eventually, the average of the three articulations was calculated. No instruction was provided regarding the loudness level during the assessment.

Statistical analyses were conducted by SPSS at the significance level of $\mathrm{P} \leq 0.05$. Kolmogorov-Smirnov test was used for determining the normal distribution of the obtained data. Multivariate Analysis of Variance (MANOVA) was used for comparing the mean scores of study groups. This test was used to examine different parameters of loudness in vowel /a/, the MPT of the vowel /a/, and loudness in reading, monologue. A repeated-measures Analysis of Variance (ANOVA) was used for comparing loudness and the MPT of the vowel /a/, loudness in reading, as well as a monologue in pre- and post-intervention in the study groups. The obtained data suggested the following items: the F values, P-values, Effect Size (ES) (partial $\eta^{2}$ ), and observed power. An ES of 0.20 was considered small, an ES of 0.50 was considered medium, and if ES was $\geq 0.80$, it was considered large [24]. The Tukey posthoc test was used for multiple comparisons of the mean values of different measurements.

\section{Results}

Table 1 presents the demographic characteristics of the study groups. The results of the MPT (sec) and the voice loudness $(\mathrm{dB})$ in the primary evaluation (pre- 


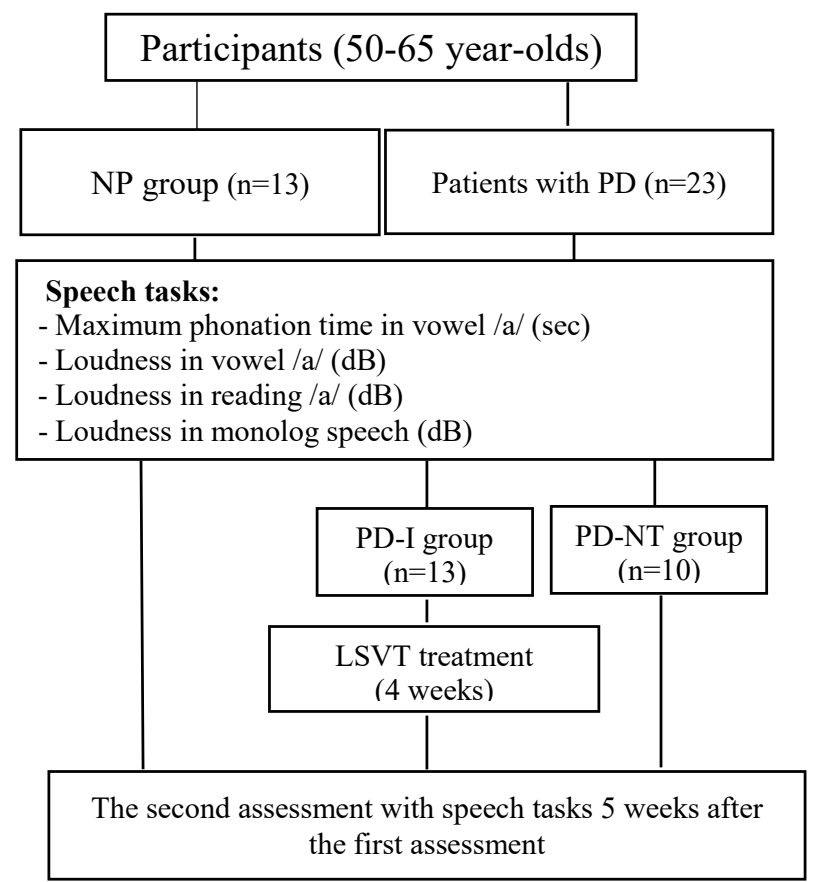

Iranian Rehabilitation Journa

Figure 1. The flowchart demonstrates the study protocol consisting of the 3 research groups, speech tasks, and the study timeline

NP: Normal Persons, PD-I: Parkinson's Disease-Intervention, PD-NI: Parkinson's Disease No-Intervention

intervention) were compared between the research groups. The PD-I and PD-NI groups were compared to the NP group, indicating significantly lower statistics in all parameters, including the MPT of the vowel /a/ $\left(\mathrm{F}_{2,33}=16.81, \mathrm{P}=0.002\right.$, partial $\eta^{2}=0.505$, power $\left.=0.999\right)$; vocal loudness in vowel $/ \mathrm{a} /\left(\mathrm{F}_{2,33}=190.76, \mathrm{P} \leq 0.001\right.$, partial $\eta^{2}=0.920$, power $=1.000$ ); vocal loudness in reading $\left(\mathrm{F}_{1,26}=113.50, \mathrm{P} \leq 0.001\right.$, partial $\eta^{2}=0.873$, power $\left.=1.000\right)$, and vocal loudness in a monologue speech $\left(\mathrm{F}_{2,33}=60.61\right.$, $\mathrm{P} \leq 0.001$, partial $\eta^{2}=0.786$, power $=1.000$ ) (Figure 2). The difference between the PD-I and PD-NI groups was not significant $(\mathrm{P} \geq 0.201)$.

After performing the LSVT intervention protocol in the intervention group, the PD-I group revealed a significant improvement in all measurements $(\mathrm{P} \leq 0.05)$. Besides, the difference between the final measurements of the PD-I and NP groups was not significant $(\mathrm{P} \geq 0.061)$. However, the PD-NI group's results in all measurements of final assessment were significantly lower than those in the PD-I $(\mathrm{P} \leq 0.001)$ and NP $(\mathrm{P} \leq 0.001)$ groups. Figure 3 compares the study groups in the MPT of the vowel /a/ $\left(\mathrm{F}_{1,26}=20.08, \mathrm{P} \leq 0.001\right.$, partial $\eta^{2}=0.549$, power $\left.=1.000\right)$; vocal loudness in vowel $/ \mathrm{a} /\left(\mathrm{F}_{1,26}=88.91, \mathrm{P} \leq 0.001\right.$, partial $\eta^{2}=0.843$, power $=1.000$ ); vocal loudness in reading $\left(\mathrm{F}_{1,26}=32.8, \mathrm{P} \leq 0.001\right.$, partial $\eta^{2}=0.666$, power $\left.=1.000\right)$, and vocal loudness in a monologue speech $\left(\mathrm{F}_{1,26}=71.83\right.$, $\mathrm{P} \leq 0.001$, partial $\eta^{2}=0.813$, power $\left.=1.000\right)$. The MPT of the vowel /a/ for the PD-I group was greater than that of the NP group; however, but this difference was not significant $(\mathrm{P}>0.078)$.

Additionally, the scores of the primary and the secondary evaluations were individually compared per group. The PD-I group demonstrated significant improvements in the second evaluation, compared with the first examination for the MPT of the vowel $/ \mathrm{a} /\left(\mathrm{F}_{1,12}=61.46\right.$, $\mathrm{P} \leq 0.001$, partial $\eta^{2}=0.861$, power $\left.=1.000\right)$; vocal loudness in vowel $/ \mathrm{a} /\left(\mathrm{F}_{1,12}=44.48, \mathrm{P} \leq 0.001\right.$, partial $\eta^{2}=0.943$, power $=1.000)$; vocal loudness in reading $\left(\mathrm{F}_{1,12}=46.30\right.$, $\mathrm{P} \leq 0.001$, partial $\eta^{2}=0.839$, power $=1.000$ ), and vocal loudness in a monologue speech $\left(\mathrm{F}_{1,12}=91.25, \mathrm{P} \leq 0.001\right.$, partial $\eta^{2}=0.976$, power $=1.000$ ). The difference was not significant between the PD-NI ( $\mathrm{P} \geq 0.055)$ and NP groups $(\mathrm{P} \geq 0.165)$ for the same analyses.

\section{Discussion}

The present study compared the effects of LSVT on the vocal loudness of Persian speakers with early PD between the PD-I and two control groups, i.e., PD-NI and NP. The main findings of this study were as follows: both study groups with PD demonstrated impaired performance, compared with the NP group in all speech tasks; the LSVT remarkably improved the vocal characteristics 

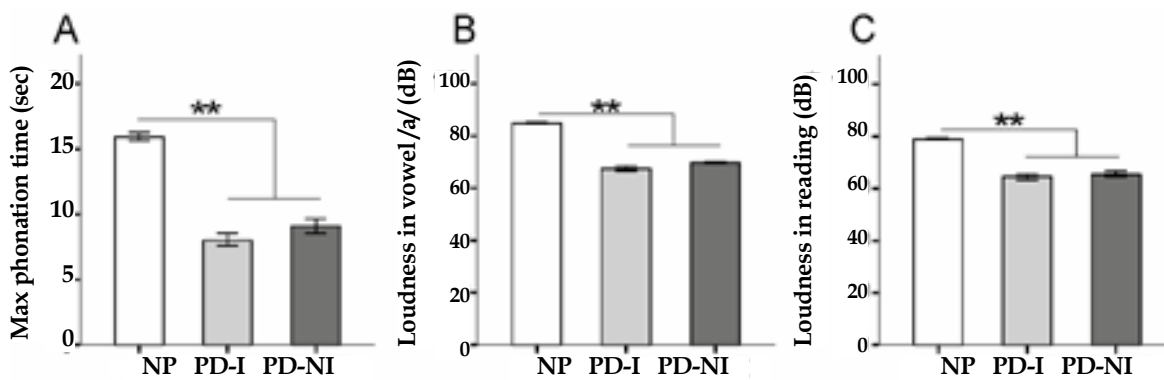

Figure 2. Comparing the data of the study groups at pre-intervention

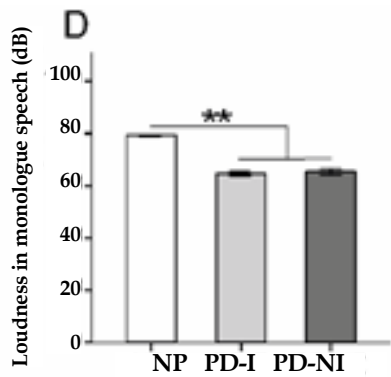

Iranian Rehabilitation Journal

A) maximum phonation time (sec), B) loudness in vowel /a/ (dB), C) loudness in reading (dB), D) loudness in monologue speech $(\mathrm{dB})$ for the three groups. NP: Normal Persons, PD-I: Parkinson's Disease-Intervention, PD-NI: Parkinson's Disease No-Intervention. Error bars represent standard error $( \pm S E)$ around the means. Asterisk indicates: ${ }^{*} \mathrm{P}<0.001$

in the PD-I group; however, no significant change was detected in the PD-NI group.

Impairments in the sustained production of vowels were tackled in some publications in patients with early PD; however, studies addressing this finding are scarce $[3,25]$. In our study, the impairment in the MPT of the vowel /a/ was presented higher in the PD groups (PD-I \& PD-NI), compared to $\mathrm{n}$ the NP group in the first assessment. The vocal-respiratory system in patients with PD reveals abnormalities in the vital capacity; the air consumed during the MPT tasks; the respiratory muscle movement, and the chest-wall movement [26]. Therefore, the MPT reduction might occur in these patients, compared with the healthy population. The MPT of the vowel /a/ in the explored patients with PD significantly increased after the LSVT; however, the NP and PD-NI groups indicated no significant difference between the primary and the secondary evaluations. Therefore, the LSVT specifically improved the MPT of the vowel /a/ in the intervention group. The LSVT intervention recalibrates the motor systems in patients with PD by increasing the movement amplitude [4]. Furthermore, the respiratory muscles' movement range might enhance. Subsequently, the MPT elevates after the intervention.
Additionally, another interesting result achieved in this study was a considerable increase in the MPT of the vowel /a/ in the PD-I group, i.e., slightly higher than the NP group. Individuals with mild PD were studied; therefore, providing training on breathing deeply (following the therapist's instructions), and persistently performing the MPT task for 4 consecutive weeks could improve their ability in this task. Besides, the PD-I group demonstrated a higher MPT, compared with the PD-NI group. Thus, the LSVT can significantly impact the disease during the early stages [4]. Further research on the early stages of the disease with mild severity, as well as investigating the persistence of the intervention effects in long-term are required.

The PD-I group also revealed a significant improvement in vocal loudness in all 3 phonations of the vowel /a/, reading, and monologue speech tasks after the intervention, compared to the PD-NI group. The present research findings were in line with those of other studies that investigated the effects of the outcomes of LSVT on increasing vocal loudness in individuals with mild to moderate and moderate PD $[4,10,11]$. Considering this compatibility and findings related to the correlation of loudness with breathing and nasalance [27, 28],
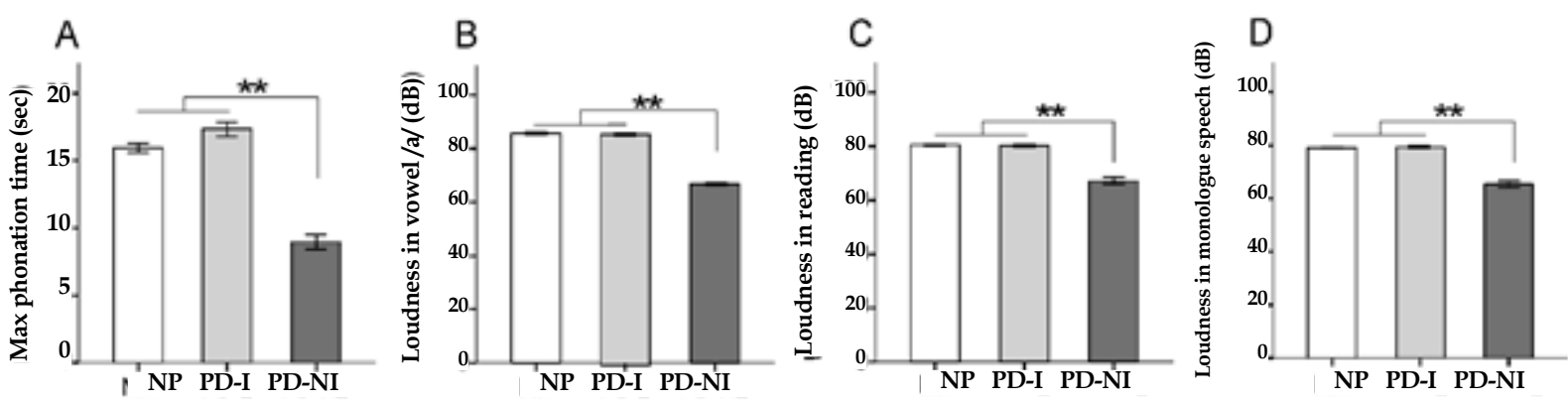

Figure 3. Comparing the obtained data of the study groups at post-intervention

Пranian Rehabilitation \ournal

A) maximum phonation time (sec), B) loudness in vowel /a/ (dB), C) loudness in reading (dB), D) loudness in monologue speech $(\mathrm{dB})$ for the three groups. NP: Normal Persons, PD-I: Parkinson's Disease-Intervention, PD-NI: Parkinson's Disease No-Intervention. Error bars represent Standard Error $( \pm S E)$ around the means. ${ }^{* *} \mathrm{P}<0.001$ 
it seems that working on the loudness of voice in these patients can be helpful. Vocal changes in the PD are also observed in the form of reduced vocal loudness, hoarseness, and breathy voice [4]; the sensory processing disorders and the inability to perceive and regulate the output amplitude are directly responsible for speech and voice disorders in Idiopathic Parkinson's Disease (IPD).

The LSVT teaches patients with PD to recalibrate their perceptive and motor systems; accordingly, their range of motor-speech parameters is less likely to reduce [8]. Besides, the LSVT overcomes or compensates for internal cueing and vocal self-monitoring deficits during the speech [12], leading to reduced sensory processing abnormalities in patients with PD-I. The LSVT is a therapeutic technique that fosters motor learning theories as well as neuroplasticity principles in motor acquisition, i.e., intensity, complexity, and saliency extension [4]. It seems that with feedback and guidance, the LSVT can engage the prefrontal cognitive circuits, as well. The effects of the LSVT on cortical regions by correcting the motor cortical, auditory, and prefrontal areas during speech has also been supported [16]. Accordingly, the LSVT could successfully induce neuroplasticity in patients with PD, leading to improvements in their vocal characteristics.

The methods used for analyzing and evaluating the vocal characteristics presented beneficial biomarkers to diagnose PD in its early stages [29]. Since $78 \%$ of patients with early PD demonstrate some voice disorders [11], the LSVT program seems crucial for mild PD. Dementia and depression are also common in these patients, which can affect speech [4]. In our study, Persian-speaking patients in the early stages of PD without dementia or depression were included; we aimed to gain more from the intervention, as well as to prevent the effects of dementia or depression on the results. Research findings presented the efficacy of rehabilitative programs for the first stages of PD; it can slow down the progression of motor symptoms [30]. In our study also the LSVT as an intervention based on motor learning theories [9] could successfully decline the progression of speech and voice problems in the first stages of PD.

The small sample size was a limitation of this study. A challenge was that the majority of the recruited patients presented advanced stages of PD; therefore, mild cases were infrequent. Besides, concerning numerous inclusion criteria, finding relevant patients for the research sample was difficult. We were also eager to follow up on the consistency of the outcomes of the LSVT program in the long term; however, the main problems of the pa- tients were resolved and they were unwilling to continue participating in the intervention program. Therefore, studying the follow-up effects of the intervention on patients at the first stages of PD is suggested.

\section{Conclusion}

The obtained data revealed that the LSVT intervention improved vocal loudness in Persian-speaking patients with early mild PD. Thus, this technique, i.e., based on the motor learning theory, can be effective in reducing the progression of the symptoms of impaired speech in the early stages of PD.

\section{Ethical Considerations}

\section{Compliance with ethical guidelines}

The Ethics Committee of Iran University of Medical Sciences approved this study (Code: 93/D/320/4189). All research participants signed informed constant forms to participating in this study. A code number was placed on each study participant's name to observe data confidentially.

\section{Funding}

This study was extracted from a $\mathrm{PhD}$ dissertation of the second author, Department of Speech and Language Pathalogy,School of Rehabilitaion Sciences, Iran University of Medical Sciences (Grant\#: 93/D/320/4189).

\section{Authors' contributions}

Conceptualization: Arezoo Saffarian, Younes Amiri Shavaki; Gholam Ali Shahidi; Methodology: Arezoo Saffarian, Younes Amiri Shavaki, Gholam Ali Shahidi, Mohammad Reza Keyhani; Investigator: Arezoo Saffarian; Data analysis: Arezoo Saffarian, Mohammad Reza Keyhani: Writing - Original Draft: Arezoo Saffarian; Writing - review \& editing, Arezoo Saffarian, Younes Amiri Shavaki, Gholam Ali Shahidi, Mohammad Reza Keyhani; Supervision: Arezoo Saffarian.

\section{Conflict of interest}

The authors declared no conflicts of interest.

\section{Acknowledgments}

We would like to acknowledge Sean Lacoursiere (CCBN) for reading and improving the manuscript. The cooperation of all participants in this study is greatly appreciated. 


\section{References}

[1] Jankovic J. Parkinson's disease: Clinical features and diagnosis. Journal of Neurology, Neurosurgery \& Psychiatry. 2008 79(4):368-76. [DOI:10.1136/jnnp.2007.131045] [PMID]

[2] Sapir S, Ramig L, Fox C. Speech and swallowing disorders in Parkinson disease. Current Opinion in Otolaryngology \& Head and Neck Surgery. 2008; 16(3):205-10. [DOI:10.1097/ MOO.0b013e3282febd3a] [PMID]

[3] Midi I, Dogan M, Koseoglu M, Can G, Sehitoglu M, Gunal D. Voice abnormalities and their relation with motor dysfunction in Parkinson's disease. Acta Neurologica Scandinavica. 2008; 117(1):26-34. [DOI: 10.1111/j.1600-0404.2007.00965.x]

[4] Sapir S, Ramig LO, Fox CM. Intensive voice treatment in Parkinson's disease: Lee Silverman voice treatment. Expert Review of Neurotherapeutics. 2011; 11(6):815-30. [DOI:10.1586/ ern.11.43] [PMID]

[5] Saffarian A, Shavaki YA, Shahidi GA, Jafari Z. Effect of parkinson disease on emotion perception using the Persian affective voices test. Journal of Voice. 2019; 33(4):580.e1-9. [DOI:10.1016/j.jvoice.2018.01.013] [PMID]

[6] Miller N, Noble E, Jones D, Burn D. Life with communication changes in Parkinson's disease. Age and Ageing. 2006, 35(3):235-9. [DOI:10.1093/ageing/afj053] [PMID]

[7] Schulz GM, Grant MK. Effects of speech therapy and pharmacologic and surgical treatments on voice and speech in Parkinson's disease: A review of the literature. Journal of Communication Disorders. 2000; 33(1):59-88. [DOI:10.1016/ S0021-9924(99)00025-8]

[8] Ramig LO, Countryman S, O'Brien C, Hoehn M, Thompson L. Intensive speech treatment for patients with Parkinson's disease: Short-and long-term comparison of two techniques. Neurology. 1996; 47(6):1496-504. [DOI:10.1212/ WNL.47.6.1496] [PMID]

[9] Ramig LO, Sapir S, Countryman S, Pawlas AA, O'Brien C, Hoehn M, et al. Intensive voice treatment (LSVT®) for patients with Parkinson's disease: A 2 year follow up. Journal of Neurology, Neurosurgery \& Psychiatry. 2001; 71(4):493-8 [DOI:10.1136/jnnp.71.4.493] [PMID] [PMCID]

[10] Ramig LO, Sapir S, Fox C, Countryman S. Changes in vocal loudness following intensive voice treatment (LSVT®) in individuals with Parkinson's disease: A comparison with untreated patients and normal age-matched controls. Movement Disorders: Official Journal of the Movement Disorder Society. 2001; 16(1):79-83. [DOI:10.1002/15318257(200101)16:13.0.CO;2-H]

[11] Sackley CM, Smith CH, Rick CE, Brady MC, Ives N, Patel S, et al. Lee Silverman Voice Treatment versus standard speech and language therapy versus control in Parkinson's disease: a pilot randomised controlled trial (PD COMM pilot). Pilot and Feasibility Studies. 2018; 4:30. [DOI:10.1186/s40814-0170222-z] [PMID] [PMCID]

[12] Sapir S, Ramig LO, Hoyt P, Countryman S, O'Brien C, Hoehn M. Speech loudness and quality 12 months after intensive voice treatment (LSVT®) for Parkinson's disease: A comparison with an alternative speech treatment. Folia Phoniatrica et Logopaedica. 2002; 54(6):296-303. [DOI:10.1159/000066148] [PMID]

[13] Cannito MP, Suiter DM, Beverly D, Chorna L, Wolf T, Pfeiffer RM. Sentence intelligibility before and after voice treatment in speakers with idiopathic Parkinson's disease. Journal of Voice. 2012; 26(2):214-9. [DOI:10.1016/j. jvoice.2011.08.014] [PMID]

[14] Martel Sauvageau V, Roy J-P, Langlois M, Macoir J. Impact of the LSVT on vowel articulation and coarticulation in Parkinson's disease. Clinical Linguistics \& Phonetics. 2015; 29(6):424-40. [DOI:10.3109/02699206.2015.1012301] [PMID]

[15] Saffarian A, Shavaki YA, Shahidi GA, Hadavi S, Jafari Z. Lee Silverman voice treatment (LSVT) mitigates voice difficulties in mild Parkinson's disease. Medical Journal of the Islamic Republic of Iran. 2019; 33:5. [DOI:10.47176/mjiri.33.5] [PMID] [PMCID]

[16] Narayana S, Fox PT, Zhang W, Franklin C, Robin DA, Vogel D, et al. Neural correlates of efficacy of voice therapy in Parkinson's disease identified by performance-correlation analysis. Human Brain Mapping. 2010; 31(2):222-36. [DOI:10.1002/hbm.20859] [PMID] [PMCID]

[17] Hughes AJ, Daniel SE, Kilford L, Lees AJ. Accuracy of clinical diagnosis of idiopathic Parkinson's disease: A clinico-pathological study of 100 cases. Journal of Neurology, Neurosurgery \& Psychiatry. 1992; 55(3):181-4. [DOI:10.1136/ jnnp.55.3.181] [PMID] [PMCID]

[18] Hoehn M, Yahr M. Parkinsonism: Onset, progression and mortality. Neurology. 1967; 17(5):427-42. [DOI:10.1212/ WNL.17.5.427] [PMID]

[19] Whitehill TL, Kwan L, Lee FP-H, Chow MM-N. Effect of LSVT on lexical tone in speakers with Parkinson's disease. Parkinson's Disease. 2011; 2011:897494. [DOI:10.4061/2011/897494] [PMID] [PMCID]

[20] Spielman J, Mahler L, Halpern A, Gilley P, Klepitskaya O, Ramig L. Intensive voice treatment (LSVT® LOUD) for Parkinson's disease following deep brain stimulation of the subthalamic nucleus. Journal of Communication Disorders. 2011; 44(6):688-700. [DOI:10.1016/j.jcomdis.2011.05.003] [PMID] [PMCID]

[21] Malakouti K, Fathollahi P, Mirabzadeh A, Salavati M Kahani S. [Validation of geriatric depression scale (GDS-15) in Iran (Persian)]. Research in Medicine. 2006; 30(4):361-9. http:// pejouhesh.sbmu.ac.ir/article-1-342-en.html

[22] Jafari Z, Esmaili M, Toufan R, Aghamollaei M. Bilingual proficiency and cognitive reserve in Persian-English bilingual older adults. Aging Clinical and Experimental Research. 2015; 27(3):351-7. [DOI:10.1007/s40520-014-0288-x] [PMID]

[23] Aghajanzadeh M, Ghorbani A, Torabinezhad F, Reza Keyhani M. Comparing of two Farsi passages in laboratory evaluation of mean and fundamental frequency variations range. Audiology. 2012; 21(1):62-8. https://avr.tums.ac.ir/index. php/avr/article/view/449

[24] Becker L. Effect size (ES). 2000. https://www. uv.es/ friasnav/EffectSizeBecker.pdf

[25] Fox CM, Ramig LO. Vocal sound pressure level and self-perception of speech and voice in men and women with idiopathic Parkinson disease. American Journal of Speech-Language Pathology. 1997; 6(2):85-94. [DOI:10.1044/1058-0360.0602.85]

[26] Solomon NP, Hixon TJ. Speech breathing in Parkinson's disease. Journal of Speech, Language, and Hearing Research. 1993; 36(2):294-310. [DOI:10.1044/jshr.3602.294] [PMID] 
[27] Huber JE. Effects of utterance length and vocal loudness on speech breathing in older adults. Respiratory physiology \& Neurobiology. 2008; 164(3):323-30. [DOI:10.1016/j. resp.2008.08.007] [PMID] [PMCID]

[28] Sadjadi V, Ghorbani A, Torabinezhad F, Amiri Y, Keyhani MR. The effect of vocal loudness on Nasalance of vowels in Persian adults. Iranian Rehabilitation Journal. 2010; 8(2):31-5. http://irj.uswr.ac.ir/article-1-189-en.html

[29] Harel BT, Cannizzaro MS, Cohen H, Reilly N, Snyder PJ. Acoustic characteristics of Parkinsonian speech: A potential biomarker of early disease progression and treatment. Journal of Neurolinguistics. 2004; 17(6):439-53. [DOI:10.1016/j. jneuroling.2004.06.001]

[30] Hirsch M, Farley B. Exercise and neuroplasticity in persons living with Parkinson's disease. European Journal of Physical and Rehabilitation Medicine. 2009; 45(2):215-29. [PMID] 
This Page Intentionally Left Blank 